\title{
Simultaneous Occurrence of Sarcoidosis and Anti-neutrophil Cytoplasmic Antibody in a Patient Presenting with Chronic Sinusitis
}

\author{
Elham Sadat Banimostafavi ${ }^{1}$, Zhila Torabizadeh ${ }^{2}$, Masoud Aliyali ${ }^{3}$, Maryam Mobini ${ }^{4 *}$ \\ ${ }^{1}$ Toxoplasmosis Research Center, Department of Radiology, Mazandaran University of Medical Sciences, Sari, Iran. ${ }^{2}$ \\ Gut and Liver Research center, Department of pathology, Mazandaran University of Medical Sciences, Sari, Iran. \\ ${ }^{3}$ Pulmonary and critical care division, Department of Internal Medicine, Mazandaran University of Medical Sciences, \\ Sari, Iran. ${ }^{4}$ Diabetes Research Center, Department of Internal Medicine, Mazandaran University of Medical Sciences, \\ Sari, Iran.
}

\begin{abstract}
Granulomatosis with polyangiitis vasculitis (GPA), formerly known as Wegener's Granulomatosis (WG), and sarcoidosis are two distinct granulomatous diseases characterized by multisystem involvement. The sequential development of these two diseases is very rare. We report a patient who initially exhibited symptoms of sarcoidosis and then limited GPA, predominantly affecting the nose and paranasal sinus, which was histologically proven. Imaging, pathological, and laboratory findings were described. After treatment, controlled clinical symptoms and changes in laboratory findings and imaging were demonstrated.
\end{abstract}

Keywords: Sarcoidosis, Granulomatosis with polyangiitis vasculitis, Vasculitis

\section{Introduction}

Granulomatosis with polyangiitis (GPA) is an autoimmune small vessel vasculitis that is highly associated with anti-neutrophil cytoplasmic antibodies (ANCA). Its clinical manifestations include necrotizing granulomatous inflammation usually involving the respiratory tract and kidney. Infectious (viral or bacterial), environmental, chemical, toxic, or pharmacological factors may trigger GPA in people who are genetically predisposed to this autoimmune disease [1]. Sarcoidosis is a multisystem granulomatous disorder with unknown etiology characterized by noncaseating granulomas in the involved organs. Diagnosis requires histological confirmation, but a presumptive diagnosis is acceptable under special circumstances [2]. Sarcoidosis and GPA are granulomatous diseases with major expressions in common sites, such as the respiratory tract, kidneys, skin, nervous system, eye and orbit, musculoskeletal system, and heart [3]. The two diseases may mimic or, rarely, follow each other [4-8]. We report an unusual case of a patient presenting with sarcoidosis that remitted with therapy who developed granulomatosis with polyangiitis vasculitis three years later.

\section{Case Presentation}

In 2017, a forty-eight-year-old woman presented with dyspnea and cough, right ankle pain, and pain in the paranasal sinuses from one month prior to her visit. The patient had a 15-year history of asthma and a 3-year history of diabetes mellitus and hyperlipidemia. Shortly after that, she was diagnosed with sarcoidosis with erythema nodosum, ankle arthritis, right paratracheal, and pretracheal lymphadenopathies (Figure 1). After one month of treatment with prednisolone and hydroxychloroquine, the patient's symptoms subsided and acute phase reactants and angiotensin-converting enzyme (ACE) levels decreased (117 $\mathrm{U} / \mathrm{L}$ to $56.4 \mathrm{U} / \mathrm{L})$.

The patient complained of epistaxis for two months in the previous year. On examination, an erythematous papular lesion in the tattoo on the left eyebrow, tenderness in the left maxillary sinus, and arthritis in the right ankle were seen. No abnormalities were detected in the heart or lung. Ophthalmologic examination showed no evidence of uveitis. Laboratory and imaging findings are shown in Table 1. Echocardiography showed no abnormal results. Lung computer tomography (CT) scan showed the disappearance of lymphadenopathy and subpleural nodule in the right lower

Personal non-commercial use only.Rheumatology Research Journal. Copyright (C) 2019. All rights reserved

*Corresponding Author: Maryam Mobini, rheumatologist, Department of Internal Medicine, Diabetes Research Center, Mazandaran University of Medical Sciences, Sari, Iran, Email: mmobini50@yahoo.com, Tel/Fax: (+98) 11-33350672, 33264044

Received: 13 February 2020; Accepted: 14 June 2020 
lobe (Figure 2). Because of the involvement of both upper and lower respiratory tracts and the disappearance of lymphadenopathy, it was decided to evaluate the patient for granulomatosis with polyangiitis vasculitis. It was noted that a high level of anti-proteinase 3 (anti PR3) and slight increase in ACE levels may be due to the patient's diabetes mellitus. Paranasal sinus CT scan showed polypoid tissue in the left maxillary sinus (Figure 3 ). A left maxillary sinus biopsy was performed in which noncaseating granu- lomas structures were observed in the epithelium with the subepithelial layer composed of aggregates of epithelioid macrophages surrounded by a collar lymphocyte (Figure 4). The patient was diagnosed with limited GPA because of the upper respiratory tract involvement without glomerulonephritis. Treatment with prednisolone and methotrexate was initiated. The patient's sinusitis symptoms were controlled and acute phase reactants and antiPR3 titers decreased (from $93 \mathrm{RU} / \mathrm{ml}$ to 30.8).

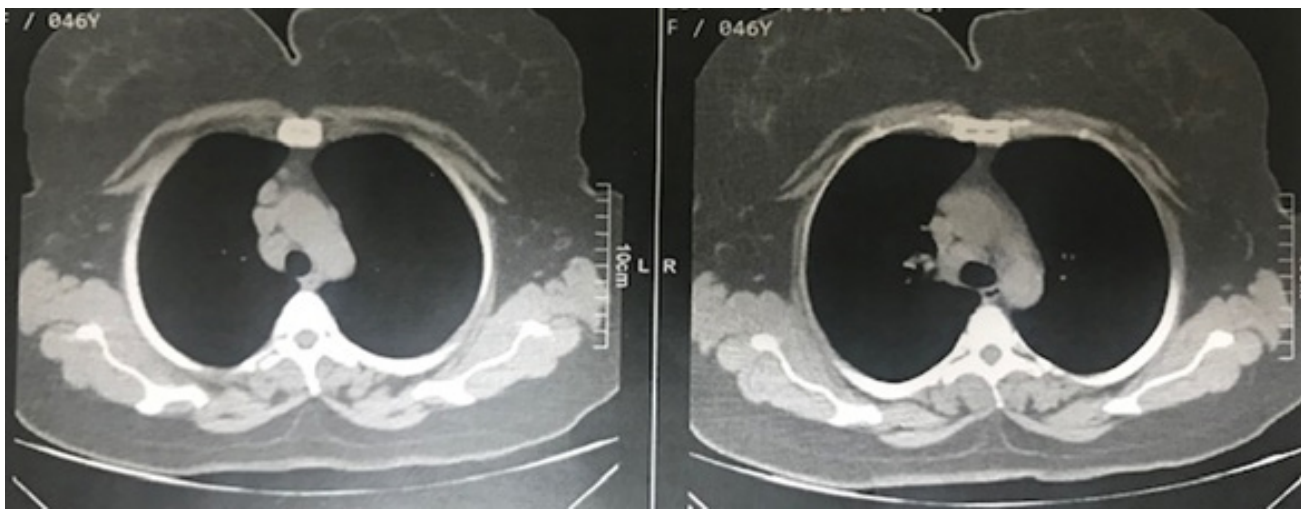

Figure 1. Computed tomography of the lung shows right paratracheal (20 mm) and pretracheal (15 mm) lymphadenopathies (2015).

Table 1. Laboratory test results of the patient

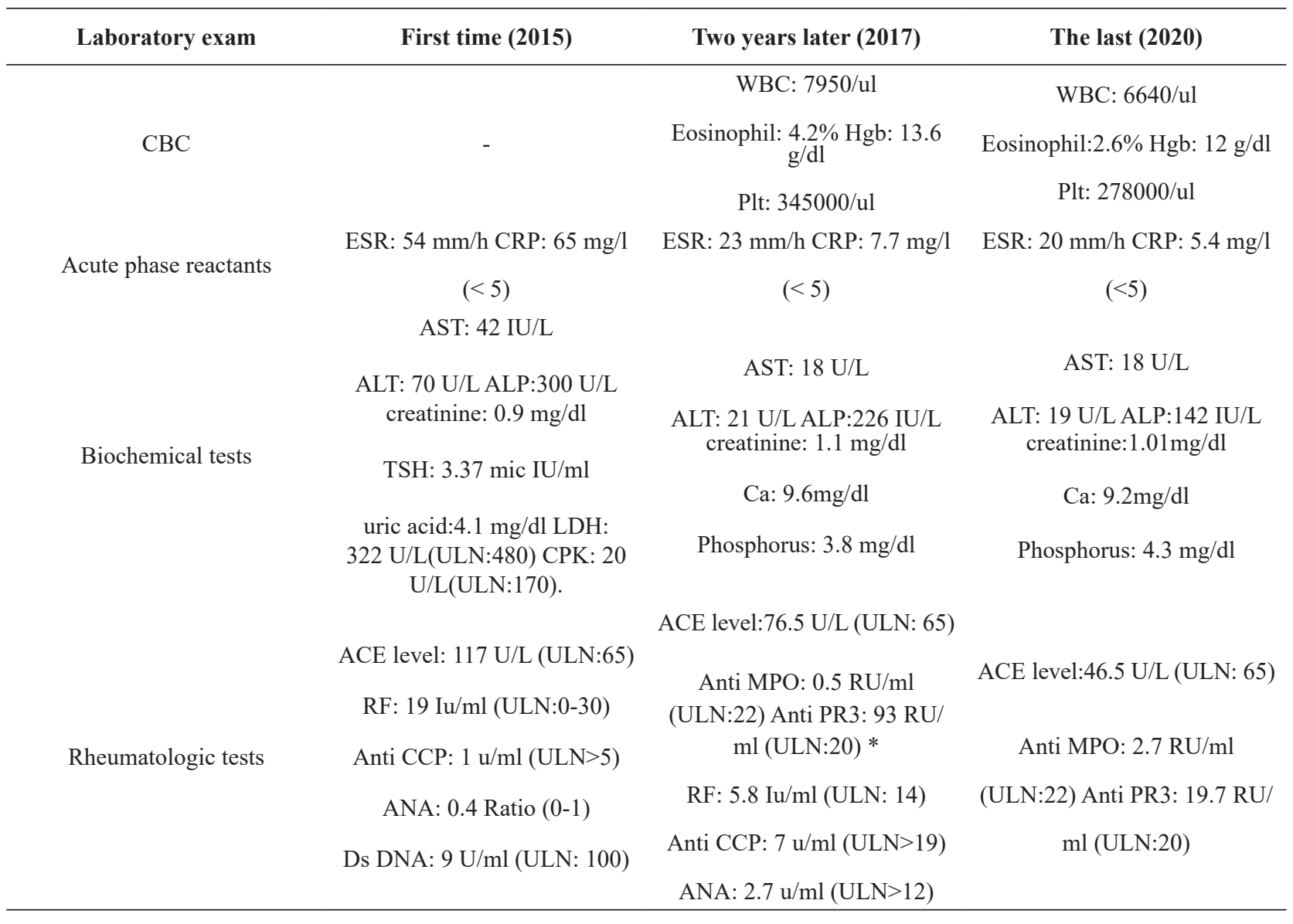




\begin{tabular}{|c|c|c|c|}
\hline Laboratory exam & First time (2015) & Two years later (2017) & The last (2020) \\
\hline \multirow{4}{*}{ Urine analysis } & WBC: $2-3$ & WBC: $5-6$ & WBC: $4-5$ \\
\hline & \multirow{2}{*}{ RBC: 0-1 } & \multirow{2}{*}{ RBC: $1-2$} & $\mathrm{RBC}: 2-3$ \\
\hline & & & Protein: trace \\
\hline & Protein: Neg & Protein: Neg & No cast \\
\hline \multirow{4}{*}{ Urine biochemistry (24 h) } & \multirow{4}{*}{-} & Volume: $750 \mathrm{cc}$ & Volume: $1600 \mathrm{cc}$ \\
\hline & & $\mathrm{Cr}: 1022 \mathrm{mg} / 24 \mathrm{~h}$ & $\mathrm{Cr}: 1211 \mathrm{mg} / 24 \mathrm{~h}$ \\
\hline & & Calcium: $228 \mathrm{mg} / 24 \mathrm{~h}$ & Calcium: $257 \mathrm{mg} / 24 \mathrm{~h}$ \\
\hline & & Protein: $57 \mathrm{mg} / 24 \mathrm{~h}$. & Protein: $75.6 \mathrm{mg} / 24 \mathrm{~h}$. \\
\hline Infectious markers & $\begin{array}{l}\text { Wright, } 2 \mathrm{ME} \text { and coombs } \\
\text { wright were all negative. }\end{array}$ & Hbs Ag: neg Anti HCV: neg & \\
\hline
\end{tabular}

*Anti PR3 was checked 3 times in different laboratories and was found to be elevated as 3-4 times higher than ULN.

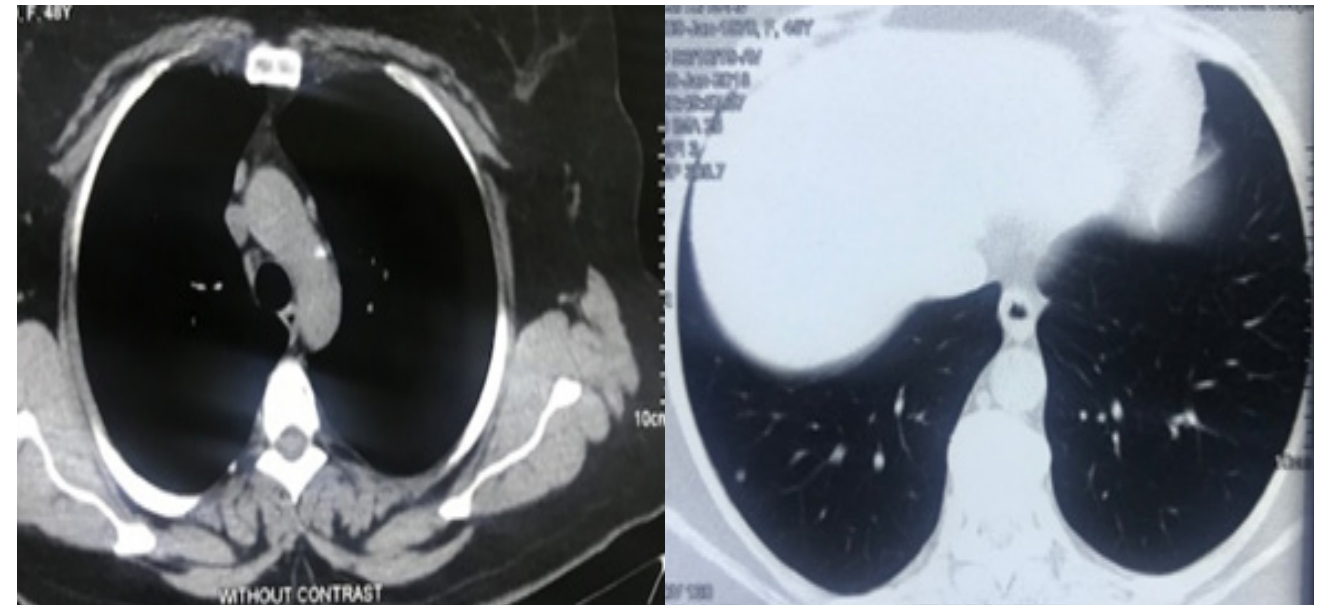

Figure 2. Left: Lung CT scan shows disappearance of lymphadenopathy (2017). Right: subpleural nodule in right lower lobe (2018).
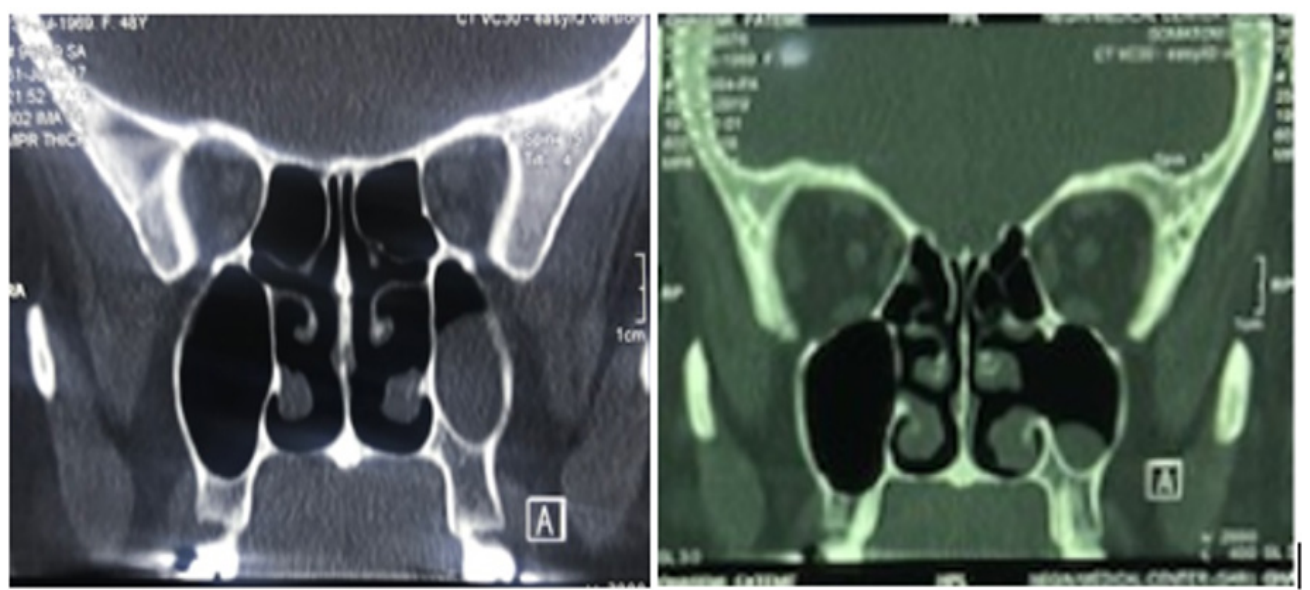

Figure 3. Left: Paranasal sinus CT scan shows polypoid tissue in left maxillary sinus (2017). Right: Size decrease in polypoid tissue in left maxillary sinus (2020). 

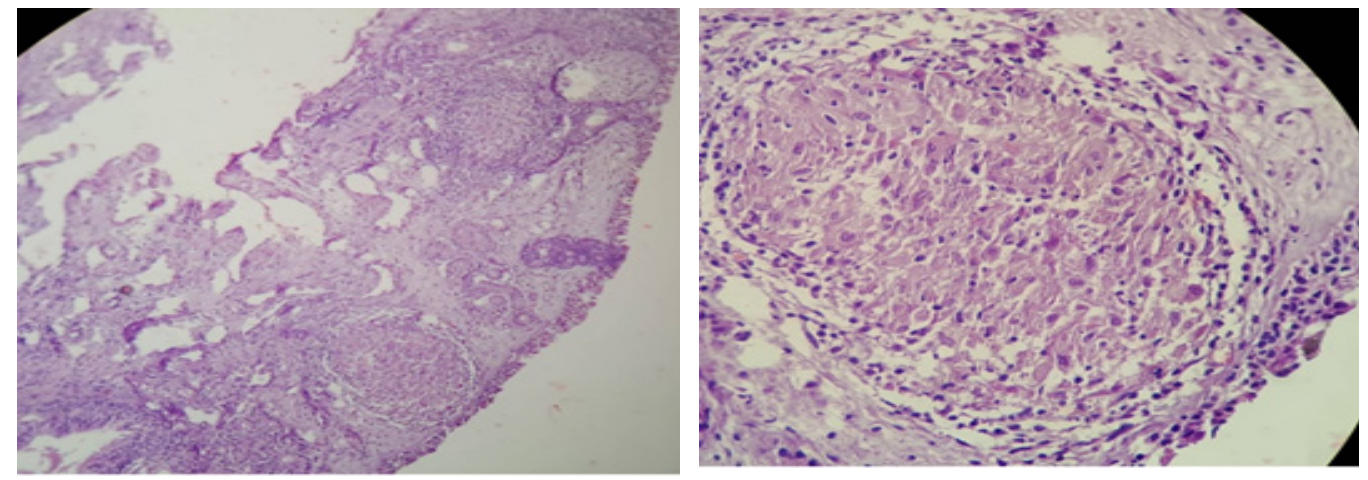

Figure 4. Respiratory epithelium with subepithelial noncaseating granulomas structures composed of aggregates of epithelioid macrophages which are surrounded by a collar lymphocyte.

\section{Discussion}

Granulomatosis with polyangiitis and sarcoidosis are granulomatous disorders with different clinical courses. In sarcoidosis, mainly T-cell mediated mechanisms are believed to be involved, whereas in GPA the presence of ANCA points toward humeral mechanisms, although T-cells may also be involved. Sarcoidosis tends to follow a more benign course in comparison with the more lethal and dramatic course of GPA [3].

Sarcoidosis may mimic or follow granulomatosis with polyangiitis vasculitis [4-8]. Nazemi et al. reported a 41-year-old man presenting with violaceous plaques, dyspnea, recurrent sinusitis, nasal bleeding, and asthma. Paraclinical data included hypercalciuria, high levels of ACE, and negative ANCA. This patient had sarcoidosis that mimicked GPA [5]. Petri et al. described a 24-year-old woman with a history of sarcoidosis with pulmonary and hepatic involvement followed by foot drop, uveitis, eosinophilia, leg ulcers, and sinus opacification. Biopsy of the leg ulcer and lung revealed numerous epithelioid noncaseating granulomas and granulomatous vasculitis. Her clinical presentation raised the possibility of GPA granulomatosis, but sarcoidosis was diagnosed because of many discrete granulomas and the lack of necrosis in her biopsies [8]. A 31-year-old woman with a two-year history of increasing nasal obstruction, crusting, and an erythematous facial rash was reported by Kuttikat et al. In this patient, ACE levels were normal, but positive PR3-ANCA was seen. The nasal mucosal biopsy showed granulomatous inflammation with vasculitis and tissue necrosis, highly suggestive of GPA. A skin biopsy also revealed histological features of sarcoidosis. The patient was diagnosed with nasal GPA and skin sarcoidosis [7].

The current case presented with epistaxis and sinusitis, high titer of antiPR3 and noncaseating granuloma structures in the epithelium with subepithelial in maxillary sinus biopsy. The patient's current manifestations do not ap- pear to be associated with sarcoidosis. The disappearance of lymphadenopathy around the trachea, absence of hypercalcemia or hypercalciuria, and normal serum ACE levels were not consistent with the recurrence of sarcoidosis. The patient met two of the criteria for a diagnosis of GPA set out by the American College of Rheumatology (ACR)and a positive antiPR3 value which, although not a criterion, has a potential value in diagnosing GPA [9]. About 90\% of patients with active generalized GPA are ANCA positive compared to limited forms of the disease (a subset in which upper respiratory tract disease predominates without renal involvement) with $60 \%$ ANCA positivity. Sensitivity of PR3 ANCA for GPA is found to be linked to the disease activity at the time of sampling [10-12]. Nasal biopsy is rarely performed in diagnostic evaluation of AAV, but it may be useful in identifying other causes of upper respiratory tract such as infection or malignancy. Upper airway biopsy may be associated with vasculitis, and the absence of vasculitis cannot rule out granulomatosis with polyangiitis vasculitis. Granulomatous inflammation is diagnostic for GPA [13].

We report an unusual case of a patient presenting with sarcoidosis that remitted with therapy who developed GPA three years later. Early diagnosis and appropriate treatment can control the disease and prevent tissue damage.

\section{Conclusion}

Sarcoidosis and granulomatosis with polyangiitis are two different systemic diseases with some similarities. The two diseases may mimic each other, or they may rarely coincide or follow one another.

\section{Acknowledgments}

The authors are grateful to the staff at Vali-Asr Hospital and all the patients who assisted with this study.

\section{Conflict of Interest}

The authors declare no conflicts of interest 


\section{References}

1. Scott DG, Watts RA. Systemic vasculitis: epidemiology, classification and environmental factors. Ann Rheum Dis 2000; 59(3):161-63. doi: 10.1136/ard.59.3.161.

2. Bargagli E, Prasse A. Sarcoidosis: a review for the internist. Intern Emerg Med 2018; 13(3):325-31. doi: 10.1007/ s11739-017-1778-6.

3. DeRemee RA. Sarcoidosis and Wegener's granulomatosis: a comparative analysis. Sarcoidosis 1994; 11(1):7-18.

4. Ahuja TS, Mattana J, Valderrama E, Sankaran R, Singhal PC, Wagner JD. Wegener's granulomatosis followed by development of sarcoidosis. Am J Kidney Dis 1996; 28(6):893-8. doi: 10.1016/s0272-6386(96)90390-x.

5. Nazemi M, Saeedi M, Yousefi M, Barikbin B, Naraghi Z, Asadi Kani Z. A case of sarcoidosis mimicking Wegener's granulomatosis. Int J Dermatol 2013; (52):1288-90. doi: 10.1111/j.1365-4632.2011.05137.x.

6. Hasni SA, Gruber BL. Sarcoidosis presenting as necrotizing sinus destruction mimicking Wegener's granulomatosis. J Rheumatol 2000; 27(2):512-14.

7. Kuttikat A, Saeed T, Chopra B, Chopra S, Chakravarty K. Nasal Wegener's and skin sarcoid--a rare combination of two granulomatous diseases. Clin Rheumatol 2006; 25(6):895-97. doi: 10.1007/s10067-005-0087-z.

8. Petri M, Barr E, Cho K, Farmer E. Overlap of granulomatous vasculitis and sarcoidosis: presentation with uveitis, eosinophilia, leg ulcers, sinusitis, and past foot drop. $\boldsymbol{J}$ Rheumatol 1988; 15(7):1171-73.

9. Leavitt RY, Fauci AS, Bloch DA, Michel BA, Hunder GG, Arend WP, et al. The American College of Rheumatology 1990 criteria for the classification of Wegener's granulomatosis. Arthritis Rheum 1990; 33(8):1101-07. doi: 10.1002/art.1780330807.

10. Bossuyt X, Cohen Tervaert JW, Arimura Y, Blockmans D, Flores-Suarez LF, Guillevin L, et al. Position paper: Revised 2017 international consensus on testing of ANCAs in granulomatosis with polyangiitis and microscopic polyangiitis. Nat Rev Rheumatol 2017; 13(11):683-92. doi: 10.1038/nrrheum.2017.140.

11. Clain JM, Hummel AM, Stone JH, Fervenza FC, Hoffman GS, Kallenberg CG, et al. Immunoglobulin (Ig)M antibodies to proteinase 3 in granulomatosis with polyangiitis and microscopic polyangiitis. Clin Exp Immunol 2017; 188(1):174-81. doi: 10.1111/cei.12925.

12. Berden A, Goceroglu A, Jayne D, Luqmani R, Rasmussen N, Bruijn JA, et al. Diagnosis and management of ANCA associated vasculitis. BMJ 2012 ;344:e26. doi: 10.1136/ bmj.e26.

13. Spanuchart I, Zungsontiporn N, P Oc, Changcharoen B, Bolger DT, Jr. Granulomatosis with Polyangiitis: A Case of Nasal Mass, Necrotic Lung, and Normal Kidneys. Hawaii J Med Public Health 2015; 74(9 Suppl 2):27-29. 